\title{
Entre la ciencia ficción y la ciencia de la historia: El corazón de la serpiente.
}

Between science fiction and the science of history: El corazón de la serpiente.

DOI: $10.32870 /$ sincronia.axxv.n79.11a21

\section{Ángel Chávez Mancilla}

Escuela Nacional de Antropología e Historia (MÉXICO)

CE: angelch.mancilla@gmail.com / ID ORCID: 0000-0002-0263-1493

\section{Esta obra está bajo una Licencia Creative Commons Atribución-NoComercial 4.0 Internacional}

Recibido: 02/06/2020

Revisado: 22/10/2020

Aprobado: $12 / 11 / 2020$

\section{RESUMEN}

En este artículo se aborda la novela de ciencia ficción El corazón de la serpiente, del soviético Iván Efremov, y se evidencia lo que identificamos como una reivindicación ideológica de la teoría marxista de la historia o materialismo histórico; y se estudia la influencia que dicho contenido ideológico tiene en la concepción de la ciencia ficción que Efremov adopta y, en un ejercicio de meta novela, expone en su mencionada obra. Para esto se expondrán algunas características de la ciencia ficción soviética en general, y las particularidades de la obra de Efremov. De igual forma se da cuenta de los aspectos esenciales de la teoría marxista de la historia, aspecto teórico en el que Efremov fundamenta la diferencia de la ciencia ficción producida en el "mundo capitalista", y su ficción del mundo futuro y el contacto extraterrestre que propone debe basarse en aspectos científicos, pues la historia como ciencia que alcanza a delinear aspectos de la futura socieda.

Palabras Clave: Ciencia ficción. Iván Efremov. Ideología. Literatura socialista. Materialismo histórico.

\section{ABSTRACT}

This article deals with the science fiction novel The Heart of the Serpent, by the Soviet Ivan Efremov, and shows what we identify as an ideological vindication of the Marxist theory of history or historical materialism; and the influence that said ideological content has on the conception of science fiction that Efremov adopts and, in a meta novel exercise, he exposes 
in his mentioned work is studied. For this, some characteristics of Soviet science fiction in general, and the particularities of Efremov's work will be exposed. In the same way, he realizes the essential aspects of Marxist theory of history, a theoretical aspect on which Efremov bases the difference of science fiction produced in the "capitalist world", and his fiction of the future world and the extraterrestrial contact that It must be based on scientific aspects, since history as a science that manages to delineate aspects of the future society.

Keywords: Science fiction. Iván Efremov. Ideology. Socialist literatura. Historical materialism.

\section{Efremov y la ciencia ficción soviética}

Aunque en los países de habla hispana aún es escaso el estudio de la ciencia ficción soviética, en los Estados Unidos hace cinco décadas que dichos estudios tuvieron gran impulso, posiblemente por las necesidades de conocer la realidad soviética durante la disputa ideológica que conllevaba la Guerra Fría y también debido a que, hacia la década de los 60’s, dicho género tuvo un nuevo auge en la Unión Soviética.

Las historias de la ciencia ficción en la URSS resaltan tres etapas. La primera hace referencia a la ciencia ficción escrita poco después de la revolución y durante el periodo de la Nueva Política Económica, momento en que el mercado de libros llegó a tener cerca de 100 títulos traducidos de literatura de ciencia ficción norteamericana y europea. (Suvin, 1971, p. 150). Posteriormente una nueva etapa se inicia en la década de 1930, considerada una época débil por motivos ideológicos atribuidos al impulso del realismo socialista de Zhdanov (Suvin, 1971; Glad, 1982; Scott, 1982; Matthew, 1988;). La tercera etapa tiene su inicio hacia mediados de la década de 1950 y se extiende durante otras dos; es considerada como periodo de revivificación de la ciencia ficción (Rullkotter, 1974; Nudelman, 1989). La obra de ciencia ficción de Iván Efremov se ubica en ésta última época, junto con los hermanos Boris y Arkadij Strugatskij, dos de los autores más representativos para la década de $1960 .^{1}$

\footnotetext{
${ }^{1}$ La obra más difundida de Boris y Arkadij Strugatski destacan es Picnic extraterrestre, este último inspiro la película Stalker de Andrei Tarkovski.
} 
La obra de Iván Efremov tuvo un gran alcance tanto en el público lector soviético como el de otras naciones; además, inspiró dentro de la Unión Soviética a un grupo de escritores de ciencia ficción Ilamado la "joven guardia", el cual se declaraba ser parte de la Escuela de Efremov. La obra más conocida de Efremov es La nebulosa de Andrómeda (1956), cuya traducción al español por la Editorial Progreso apareció en (1965); luego están otras como: El país de la Espuma, La hora del toro de (1968), Naves de estrellas (1948), y El corazón de la serpiente (s/f). ${ }^{2}$

Las novelas de Efremov, además de ser ineludibles en los estudios generales de la ciencia ficción soviética, también han sido objeto de múltiples estudios específicos. ${ }^{3}$ Sumado a esto, en las últimas décadas dentro de los estudios sobre ciencia ficción soviética existe una tendencia a destacar los aspectos sobrenaturales, ocultistas y milenarios que subyacen a la trama, así como la posible disidencia política de los autores respecto del gobierno Soviético, y la obra de Efremov también ha sido analizada bajo este enfoque.

Así pues, los análisis de la ciencia ficción soviética han dejado de lado el análisis de lo que parecería el aspecto más obvio de dicha literatura: la carga ideológica socialista y la posible influencia del marxismo. Esto puede deberse a que se da por hecho que en la literatura soviética se encuentran aspectos del realismo socialista, ello pese a las temáticas propias del subgénero literario (Brandis, 1950). No obstante, el aspecto ideológico que más se resaltó fue el del realismo socialista, cuyas pautas fueron dadas por Andrei Zhdánod en el Primer Congreso de Escritores Soviéticos en agosto de 1934.

Un ejemplo del resultado de vincular el realismo socialista con otros aspectos de la realidad soviética fue el siguiente:

Por un lado, en términos de realismo socialista, se tenían que popularizar futuros desarrollos sociales y especialmente científicos en el espíritu de la construcción socialista,

\footnotetext{
${ }^{2}$ El corazón de la serpiente se editó en ruso en 1959, no obstante, la edición en español que se empleará en el presente artículo no cuanta con registro de año de edición, por lo que se utilizará el año de su publicación en idioma original, esto por considerar que contribuye a ubicar la obra en cuestión.

${ }^{3}$ Véase, por ejemplo: Grebens (1978), Ivan Efremov's Theory of Soviet Science Fiction. Nueva York: Vantage Press; Brandis y Dmitrevskii (1963), Cherez gory vremeni. Ocherk tvorchestva I. Efremova. Moscow.
} 
mientras que, por otro lado, también se esperaba que anticiparía desarrollos futuros en términos de Maxim Gorky's definición de mito y folklore. (Schwartz, 2011, p. 216). ${ }^{4}$

También se ha estudiado cómo el realismo socialista se combinó con el desarrollo científico e industrial soviético para expresarse en la ciencia ficción (Tucker, 1974, p. 194). Otro aspecto ideológico estudiado es la lucha entre el socialismo y el capitalismo; parte de la teoría del marxismo soviético y posteriormente del realismo socialista, se expresó desde las primeras obras de la ciencia ficción elaboradas posteriores a la Revolución de Octubre con la constante crítica al "viejo capitalismo en Europa y América". (Suvin, 1971, p. 5).

Sin embargo, el realismo socialista no es fue el único aspecto ideológico de corte marxista que influyó en la ciencia ficción soviética, aunque los análisis se han centrado principalmente en éste y pocos han destacado la presencia de otros aspectos del marxismo. Un ejemplo de esto es el estudiado (Nudelman, 1989). Por tanto, pese al ostensible reconocimiento de que la ciencia ficción soviética puede estar cargada de aspectos ideológicos del marxismo, poco han avanzado los estudios en esta dirección, tal vez porque para poder situar la existencia de postulados de la teoría marxistas dentro de las obras de ciencia ficción soviética se requiere el conociemito y familiaridad con la teoría marxista ortodoxa que se difundió en la Unión Soviética.

De igual forma, poco se ha adelantado en el estudio del tipo de relación entre la ciencia y hombre que se expresa en la ciencia ficción soviética, aspecto que es posible abordar dado que la ciencia ficción al discurrir sobre posibles aspectos futuros de la ciencia también reflexiona sobre las implicaciones de las aplicaciones sociales de nuevos conocimientos técnico-científicos. Al proyectar la sociedad futura, la literatura soviética consideraba la existencia del comunismo en el planeta tierra, por lo que se destacaba que la relación entre ciencia y hombre debía ser armónica: “En la sociedad socialista, el crecimiento de la ciencia y la técnica, cualquiera que sea su envergadura, es el crecimiento de las fuerzas propias del hombre" (Vólkov, 1975, p.8).

\footnotetext{
${ }^{4}$ La traducción del inglés de esto texto y del resto de citas de artículos en inglés fue hecha el autor del artículo que se propone para su publicación.
} 
Esto se diferencia de la relación entre hombre y ciencia dentro del capitalismo, donde, según el autor soviético Volkov:

[...] el hombre pierde la noción de que la ciencia y la técnica son producto de su propia actividad y les asigna vida independiente y poder místico sobre la gente, llegando a ser encarnación de las relaciones que dominan al hombre y que lo estropean física y moralmente. (1975, p14).

Es decir, la relación entre hombre y ciencia implica un proceso de enajenación que puede llevar a la maquinofobia o a deificar a la conciencia. Para los soviéticos esto era el fundamento que llevaba a que la ciencia ficción norteamericana, al imaginar la sociedad futura y la repercusión de las nuevas tecnologías sobre esta, se concretara en la producción de distopías (Jameson, 1982, p.153).

Frente a la distopía de la ciencia ficción norteamericana y del mundo capitalista, en el Unión Soviética este género literario dio por resultado la utopía, en parte debido a la carga ideológica que debía mostrar el socialismo-comunismo como un sistema social superior a capitalismo, de ahí que Frederic Jameson afirme que "lo que es ideológico para el lector soviético puede ser utópico para nosotros" (1982, p.155). Sin embargo, el hecho de que en la ciencia ficción en el mundo capitalista expresara frutos en los que el hombre entra en conflicto con las máquinas, donde la humanidad entra en conflicto con los seres extraterrestres, también es parte de la extrapolación de la realidad capitalista a la literatura, es decir, tanto la ciencia ficción del capitalismo como la producida en el mundo socialista se pueden apreciar aspectos ideológicos consientes o inconscientes.

En la forma como los escritores conciben el futuro, la relación entre la humanidad y la ciencia, la humanidad y los seres extraterrestres, se encuentran otros de los aspectos ideológicos marxistas. En ese sentido, no es casual que en la literatura soviética:

[...]los extraterrestres fueron concebidos como amigos y no como invasores hostiles, no como colonizadores con la intención de permanecer en la tierra, sino más bien como visitantes temporales, que posiblemente tuvieron una influencia positiva en la historia humana y siempre señalaron la esperanza para el futuro. (Schwartz, 235). 
Así pues, en el futuro imaginado por los literatos soviéticos podían expresarse dos aspectos ideológicos: 1. El interés de presentar al socialismo como un sistema social que ofrecía más beneficios a la humanidad que el capitalismo; 2 . La ideología marxista que sostiene que el tránsito de la sociedad hacia el socialismo estaba basado en leyes histórico-sociales, o como dice Rafail Nudelman, la ciencia ficción como expresión ideológica puede considerarse como un "Modelo cognitivo de cambios reales y de una ideología en particular" (Nudelman, 1989, p.38). El primer aspecto ya ha sido estudiado para algunas obras; respecto del segundo aspecto analizaremos su presencia en El corazón de la serpiente (s/f) de Iván Efremov. ${ }^{5}$

\section{La ciencia de la historia}

El marxismo ortodoxo considera que la disciplina de la historia es una ciencia, esto lo fundamenta en la teoría de la historia o materialismo histórico. Por ejemplo, el manual soviético de teoría de la historia más difundido en la URSS enuncia:

El materialismo histórico es la ciencia de las leyes más generales que rigen el desarrollo de las sociedades [...] versa sobre las leyes del desarrollo de la sociedad en su conjunto, sobre las relaciones mutuas sobre todos los aspectos de la vida social. (Konstantinov, 1956, p.1).

Afirmaciones similares que postulan a la historia como ciencia se pueden encontrar las obras de autores como Evgueni Zhúkov (1982) y Ernesto Schettino (1981).

El carácter de ciencia que el marxismo ortodoxo da a la historia y las disciplinas afines no fue una cosa menor dentro de la sociedad soviética. El testimonio del historiador y arqueólogo Gordon Childe en si visita a la URSS lo deja más claro:

La arqueología y la antropología están clasificadas como ciencias en la URSS, y junto con otras ciencias sociales figuran bajo la égida de la Academia de Ciencias del mismo modo que

\footnotetext{
${ }^{5}$ La edición en español que usamos para el presente artículo no cuenta con año de impresión, pero para identificar ésta obra usaremos el año de 1959 en que se dio la publicación del libro en ruso.
} 
las ciencias naturales. De aquí que durante la guerra los arqueólogos y antropólogos hayan sido "reservados" al igual que los geólogos y los físicos. (Bunge, 1947, p.61).

Al considerar a la historia como ciencia también se le atribuían características como el tener leyes generales $y$, por tanto, la posibilidad ser de utilidad para la predicción de la tenencia de acontecimientos futuros (Schettino, 1993). Entre los aspectos históricos que el marxismo predice a futuro está la sustitución del sistema capitalista por el socialismo-comunismo, de aquí que la ciencia ficción soviética postulara el desarrollo humano futuro como una sociedad comunista. Para comprender de mejor forma las implicaciones teóricas de esta proyección se deben tener en cuenta las principales leyes histórico-sociales: Ley del papel determinante del modo de producción en la sociedad; Ley de la correspondencia entre fuerzas productivas y relaciones de producción para el paso de un modo de producción a otro; Ley de la relación y mutua determinación entre la base y la superestructura. La esencia de estas leyes es expuesta por Marx en el "Prólogo a la contribución de la crítica de la economía política" (Marx, 1970, pp37-43).

La referencia a los modos de producción fue una constante en la ciencia ficción soviética. En la temprana obra de Alexander Bogdanov, Estrella roja (2010) se plantea que en Marte la sociedad ha llegado a un modo de producción socialista. Por otra parte, la novela Qué difícil es ser Dios de Arkadi y Boris Strugatski (1970) plantea el contacto de los humanos de una sociedad comunista con seres de otro planeta cuyo desarrollo social tiene las características del modo de producción feudal. Aunque Nudelman ha observado que la ciencia ficción de la tercera etapa "proporcionó a los lectores un método y un modelo para comprender la historia" $(1989$, p.50), en sus análisis no avanza a profundizar en los rasgos de la concepción materialista de la historia que se expresan en dichas obras, que es la teoría de los modos de producción.

Nudelman afirma que "Los aspectos de la realidad histórico-social de la tierra fueron llevados al espacio, esto lleva a que se hable de revoluciones planetarias" (1989, pp.40-41). Sin embargo, aunque el aspecto más difundido de la teoría marxista es la lucha de clases, en el "Prólogo a la contribución de la crítica de la economía política", Marx expone los elementos centrales de su 
concepción del desarrollo histórico y omite la lucha de clases como móvil de la historia, pero sí hace referencia a la Ley de la correspondencia entre fuerzas productivas y relaciones de producción, que explica la sustitución de un modo de producción por otro y es la ley histórica más general la que prevé la inminente llegada del socialismo.

La otra ley del materialismo histórico es la de la relación y mutua determinación entre la base y la superestructura, que establece que la base material de la sociedad determina, en última instancia, el aspecto ideológico; establece que la base material de la sociedad determina aspectos del pensamiento.

Este aspecto ya hemos mencionado que fue la base para que los soviéticos, basados en su concepción de la sociedad, establecieran que el contacto con extraterrestres sería pacífico; reflejaban en su concepción de la sociedad, no una de conflicto, expansión, guerra ni explotación. Esta ley de la historia aplicada al análisis de la ciencia ficción soviética explicaría el motivo de que la ciencia ficción norteamericana expresara futuros de conflicto interplanetario.

Para llegar a esta reflexión sobre la presencia de aspectos de la teoría marxista en la ciencia ficción, tuvo una expresión temprana no en un crítico literario sino en el escritor Ivan Efremov, que en su novela El corazón de la serpiente, plasma por medio de sus personajes una reflexión de aspectos ideológicos los principios del materialismo histórico. Recordemos que Efremov, una vez que se convierte en investigador de paleontología, tuvo contacto con la concepción marxista soviética de las ciencias naturales y sociales, por lo que no es de extrañarse que conociera aspectos del materialismo histórico; además de que el materialismo histórico era la visión oficial de la historia que se enseñaba en la Unión Soviética.

Un ejemplo de las reflexiones de sus personajes que explicitan aspectos de la teoría marxista de la historia lo da Mut Ang, el capitán de la nave Telurio que lleva a la expedición humana. Este personaje, al hablar sobre la importancia de la labor de investigación que hace la expedición, y al referirse al desarrollo del conocimiento y de la sociedad, destaca el desarrollo dialéctico en espiral: "cada siglo de su historia, la humanidad retrocedió en algo, a pesar de su ascenso general conforme 
a la ley del desarrollo en espiral. Cada siglo tuvo sus particularidades y sus rasgos comunes..." (Efremov, 1959, p.18).

El mismo personaje da otro ejemplo la disertar sobre cómo los hombres del "mundo antiguo" concebían la historia. En este caso destaca la visión progresista del cambio a futuro de la sociedad socialista salida del capitalismo y no la mirada al pasado o concepción reaccionaria: "La gente buscaba involuntariamente lo bueno en el pasado, soñando con su repetición, y tan solo los espíritus fuertes habían sabido prever e intuir la inevitable mejoría en la organización de la vida humana que les brindaba el futuro" (1959, p.50). Este fragmento, sin hacer referencia a ellos, explicita a los teóricos del marxismo: les dirige un panegírico. En la URSS se daba el debate contra los populistas y el romanticismo económico (Lenin, 1970), pero es un aspecto que Marx y Engels ya expresaban en el Manifiesto del partido comunista al hablar del socialismo utópico o socialismo reaccionario (1972, pp.81-94).

Pero el aspecto que más destaca Efremov en esta novela es la relación entre base y superestructura, y lo hace partiendo de la idea de que las producciones artístico-literarias, al ser una forma de ciencia, responden a la base material de la sociedad. Al proponer esta reflexión, también presenta un ejercicio de meta novela, pues en su novela los personajes de ciencia ficción reflexionan sobre la escritura de ella. Esto se expresa de la manera siguiente: en la sociedad comunista ya se ha desarrollado plenamente la humanidad, todos los aspectos idealistas basados en la ignorancia han desparecido: "En las novelas antiguas aparecían siempre ruinas misteriosas, profundidades ignotas, alturas inaccesibles, y antes aún, arboledas, fuentes trochas y asas encantadas, malditas, dotadas de fuerzas mágicas" (Efremov, 1959, p.30). También Mut Ang, reflexionado sobre la historia, piensa: “En las novelas históricas todo está descrito de forma tan viva y amena [...] iLa de veces que se había comentado y descrito, en las viejas novelas, la soledad del hombre caído en el futuro! [...]" (p. 41).

La reflexión sobre la novela se enriquece más cuando trata sobre las novelas de ciencia ficción. Nuevamente es por medio de Mut Ang que se dirige a la tripulante Kari Ram: 
¿Y sabe cómo nuestros antepasados se representaban, ya en la época inicial del asalto al espacio, el primer encuentro con los habitantes de otros mundos? Como un choque bélico en el que las naves se destruían brutalmente y los hombres se aniquilaron los unos a los otros. [...] Nuestros literatos modernos no escriben nada del tenebroso periodo de decadencia del capitalismo. Pero ustedes saben, por los manuales de historia utilizados en la escuela, que nuestra humanidad atravesó en su tiempo un periodo bastante crítico de desarrollo. (1959, pp.47-48).

Por sí mismo, este fragmento contiene aspectos como referencias al modo de producción, y, como hemos dicho, a la explicación de que, bajo la base económica capitalista, la forma de pensar del futuro estaba relacionado con elementos bélicos. También se debe notar su referencia a que la historia era conocida por manuales en la escuela, porque los literatos de la sociedad futura no escribían sobre la "decadencia del capitalismo". El fragmento citado permite a Efremov enlazar la reflexión sobre la novela a la reflexión sobre la teoría de la historia materialista, lo que le permite discurrir sobre las posibilidades de que la imaginación de la sociedad futura esté basada en la ciencia de la historia

Estos aspectos de la teoría materialista de la historia son reforzados por el argumento de otro personaje, Kari Ram:

Cuando los seres humanos habían aprendido ya a dominar la materia y el espacio, las relaciones sociales conservaban aún sus viejas formas y el desarrollo de la conciencia social se hallaba retrasado también respecto a los adelantos de la ciencia. (Efremov, 1959, p.48).

Para entender mejor la referencia de este aspecto recordemos lo que dice Marx en su mencionado Prólogo:

Al llegar a una determinada fase de desarrollo, las fuerzas productivas materiales de la sociedad chocan con las relaciones de producción existentes [...] De las formas de desarrollo de las fuerzas productivas, estas relaciones se convierten en trabas suyas. Y se abre así una 
época de revolución social. Al cambiar la base económica, se revoluciona más o menos rápidamente, toda la superestructura erigida sobre ella. (1970, p.40).

Cuando Efremov habla del dominio de la materia y el espacio está retratando el alto grado de desarrollo de las fuerzas productivas, y abiertamente menciona que las relaciones sociales estaban retrasadas, es decir, habla de la época de revolución social. También hace referencia a que la conciencia de la sociedad estaba retazada respecto de las fuerzas productivas, y esto también es recuperar la idea central del texto citado de Marx, pues explica que la conciencia esta retrasada dado que se requería cambiar la estructura o base económica, esto es, hacer resolver la contradicción entre fuerzas productivas y relaciones de producción desapareciendo la existencia de la propiedad privada de los medios de producción. Pero esta conciencia atrasada también es abordada en el prólogo de Marx, al decir que en el estudio de las épocas de revolución social se deben considerar los cambios materiales y los de corte ideológico. Recordemos que, para la visión marxista, la literatura es una expresión ideológica, una expresión de la conciencia de la sociedad, y al respecto Marx menciona:

Y del mismo modo que no podemos juzgar a un individuo por lo que piensa por sí, no podemos juzgar tampoco a estas épocas de revolución por su conciencia, sino que, por el contrario, hay que explicarse esta conciencia por las contradicciones de la vida material y por el conflicto entre las fuerzas productivas y las relaciones de producción. (1970, p.40).

Para el marxismo una expresión de la conciencia de la época de revolución social estaría materializada en las novelas de ciencia ficción, y se pensaría que la que expresan es poco fiable, pues responden a esa conciencia atrasada respecto del desarrollo material. Esto no se aplica a todas las novelas de ciencia ficción, sino a las capitalistas, o aquellas que no pongan atención a la contradicción de fuerzas productivas/relaciones de producción. En este entendido, las novelas de corte soviético si atenderían a una visión más fiel de la realidad. Esto plantea al mismo tiempo la problemática sobre si es posible afirmar que hay novelas cuya ficción de la sociedad futura pudiera 
estar más apegada a la ciencia de la historia y otras que no, lo que llevaría a pensar en la posible postulación de novelas de ciencia ficción que sean duras y blandas respecto de los postulados de la ciencia de la historia.

Volviendo al hilo expositivo de la teoría materialista de la historia, que hasta aquí consideramos va de la mano del "Prólogo a la contribución de la crítica..." de Marx, Efremov, buscando ser lo más preciso posible, hace que Mut Ang replique a Kari Ram:

La definición es casi exacta. Usted tiene buena memoria, Kari. Pero formulémoslo, en otros términos: El conocimiento y dominio del universo chocaron con la primitiva mentalidad del propietario individual. El futuro y la salud de la humanidad se hallaban puestos en platillo de la balanza del destino años antes de que triunfara el progreso y de que el género humano formara una sola familia en una sociedad sin clases. En la mitad capitalista del mundo, la gente no veía nuevas vías de desarrollo y consideraba su formación social como algo eterno e inmutable, que degeneraría en el futuro, en guerras inevitables y suicidas. (Efremov, 1950, p.48-49).

Nuevamente se afirma que el pensamiento atrasado se corresponde con las relaciones capitalistas del "propietario individual", y esto entró en choque con el desarrollo de las fuerzas productivas, es decir con el "conocimiento y dominio del universo". Este fragmento parece traducir los aspectos teóricos a una forma más accesible, o que le da un carácter pedagógico. También se puede destacar que Efremov acusa al mundo capitalista de concebir la sociedad como inmutable, aspecto que hacia el fin de la guerra fría se plasmaría de forma teórica en El fin de la historia de Francis Fukuyama, y por tanto concebir que la guerra es un aspecto inherente a la sociedad. Así pues, se refrendan la ley de la correspondencia entre fuerzas productivas y relaciones de producción, y la ley de la determinación de la base material sobre la estructura ideológica.

Una vez explicitados los aspectos de teoría de la historia en los que se basa Efremov, se comprende mejor la reflexión que hace de la novela de ciencia ficción como una expresión característica de la ideología ya sea del sistema capitalista o socialista. Para hablar sobre la ciencia ficción en el mundo capitalista, en voz de Mut Ang expresa: "Tengo en mi bibliofilmoteca de 
películas históricas un libro muy característico de aquellos tiempos..." (1959, p.50). El texto al que hace referencia explícita Efremov por medio de su personaje (1959, p.69) es Primer contacto (1945), un escrito de ciencia ficción del norteamericano Murray Leinster. El vínculo entre El corazón de la serpiente y Primer contacto ya ha sido señalado por otros autores, (Barrett, 2012; Suvin, 1971, p.115), no obstante, poco se ha ahondado en la significación teórica de dicha regencia que le permite a Efremov disertar sobre el género de la ciencia ficción y el materialismo histórico.

La reflexión que Efremov presenta del texto mencionado es que ahí:

El encuentro en el espacio significaría únicamente comercio o guerra. Nada más cabía en la mente del autor. [...] Puso en claro que el capitán de la otra nave, guiado por conocimientos sociales tan primitivos como los de la gente de la Tierra, había estado devanándose los sesos ante el problema de cómo escapar vivos de aquella espantosa situación, sin destruir la nave de la Tierra. (1959, p.70).

En este fragmento, la crítica fundamental que dirige Efremov al texto de Murray Leinster es referente a que éste concibe la guerra como una forma eterna y universal de relacionamiento social, pues tanto la nave extraterrestre como la terrestre, aun siendo el primer contacto, entran en un momento de tensión al considerar que lo más probable es ser agredidos, por el contrario.

Esto contrasta con Thomas Barrett, quien considera que Leinster tuvo una posición casi neutral durante la guerra fría pues "no proporcionó críticas izquierdistas de la sociedad estadounidense ni alarmas conservadoras contra las amenazas soviéticas" (Barret, 2012, p.95-96). Esto no implica que no tuviera una posición ideológica consciente o inconsciente, de hecho, llega a considerar que la acusación que Efremov hace de belicista a Primer contacto es incorrecta porque, a diferencia de otros textos de ciencia ficción donde sí se da la guerra entre seres de distintos planetas, en Primer contacto esto no ocurre. A ojos de Efremov, la concepción belicista está presente desde el momento en que se plantea la posibilidad de beligerancia entre sociedades que han conquistado el espacio. De esta forma nuevamente Efremov destaca la relación estructura superestructura, pues atribuye al sistema económico capitalista "la razón de la ideología bélica, la 
propaganda de la idea de que las guerras son inevitables y eternas en el Cosmos" (s/f, p.78), y añade que esta es la quinta esencia del mal en el corazón de la serpiente.

Para Efremov, quien escribía desde una concepción socialista, el primer contacto entre seres racionales de distintas plantas no podría ser la guerra o agresión, pues se sobre entendía que tanto los humanos como los otros seres debían perecer a una sociedad comunista:

La humanidad no puede vencer el espacio mientras no haya adoptado el modo superior de vida en el que no hay lugar para la guerra y en el que cada cual se siente responsable por todos. [...] La humanidad no era capaz de vencer las fuerzas de la naturaleza en el plano cósmico antes de haber ascendido al grao superior de la sociedad comunista. (1959, p.81).

Aunque se ha estudiado a El corazón de la serpiente como una respuesta a Primer contacto, es posible considerar que el texto de Leinster sea solo un ejemplo, y que la aspiración real de Efremov fuera hacer una crítica generalizada a la ciencia ficción del mundo capitalista. Esto se sustenta en que, al apoyarse en la teoría marxista de la base y la superestructura, estaría generalizando las críticas de Primer contacto para el conjunto de la ciencia ficción elaborada en las naciones capitalistas.

Además de señalar que la ciencia ficción capitalista tiene una base ideológica no científica, Efremov postula que es posible otra forma de imaginar el futuro, y que ésta podría estar basada en las leyes de la ciencia de la historia. Es confesión de parte de Efremov que afirma que su novela fue pensada con base en la ciencia de la historia. Esta reflexión sobre la posibilidad de imaginar la sociedad futura con base en la ciencia de la historia llevaría a plantear la siguiente problemática para la ciencia ficción: ¿la ciencia ficción basada en la ciencia de la historia pierde un tajo de ficción? No puede perder la esencia ficcional, pero la ciencia de la historia daría un marco referencial, además de que, aun aceptando a la historia como ciencia, ésta no podría sino darnos trazos generales de leyes generales y no especificidades. 


\section{Los otros seres racionales}

De igual forma que Efremov se vale de la teoría materialista de la historia como base para imaginar el futuro, también se apoya en otros aspectos del marxismo de corte soviético para dar una base más real a la forma que podían tener los alienígenas. Aquí el autor sigue de cerca a la teoría marxista en su definición de conciencia como "producto de la materia, fruto de su desenvolvimiento, es decir, una propiedad de la materia altamente organizada" (Arjiptsev, 1966, p. 131), que implicaba una relación entre la conciencia y la fisiología humana (Shorojova, 1979), lo que implicaría que la conciencia y el cerebro, órgano que permite la existencia de la conciencia, están interrelacionados. Efremov expresa esto por medio de las reflexiones que sus personajes acerca de cómo podrían ser esos otros seres racionales:

[...] la humanidad había rechazado hace tiempo las teorías, ahora muy en boga, de que los seres racionales podían existir bajo las formas y estructuras orgánicas más diversas. [...] En realidad, la anatomía y fisiología del hombre -único ser de la Tierra dotado de cerebro capaz de razonar- no eran un capricho de la naturaleza, sino que representaban el grado máximo de adaptación al medio ambiente y correspondían a la capacidad del cerebro y de todo el sistema nervioso desarrollar una gran actividad. (1959, p.62).

La conclusión de los personajes de Efremov deriva de la idea de que "Todo ser racional de otro mundo, que explore el Universo, debe ser, por este propio hecho, tan perfecto y universal como los hombres de la tierra y, por lo tanto, igual de hermoso" $(1959$, p.62). Con estas reflexiones basadas en el materialismo dialéctico o filosofía marxista de corte soviético, se plantean a los escritos de ciencia ficción un marco para poder discurrir con una base teórica acerca de las formas de los seres racionales de otros planetas.

Esto explica por qué cuando en los personajes humanos de Efremov entran en contacto con los otros seres, estos son descritos con una corporeidad muy similar a la humana, al grado que "Entre ellos había, evidentemente, mujeres, a juzgar por sus cuellos largos y ben formados, por la redondez de sus facciones y por sus melenas cortas y muy espesas. Los hombres eran más altos y 
más fornidos, tenían más ancho el mentón y, en general, las mismas particularidades que les diferenciaban del sexo opuesto en la tierra" (1959, p. 62).

Así pues, para Efremov no solo las leyes histórico-sociales del marxismo tiene carácter universal, sino también las concepciones filosóficas respecto a la conciencia y la materia tienen un carácter universal que permite prever algunos aspectos materiales de los seres racionales de otros planetas. Efremov, basa su fantasía de sociedades y seres racionales de otros planetas en la ideología marxista, con lo que busca distinguirse de la ciencia ficción que no toma la teoría marxista como guía.

\section{Conclusiones}

Pese a que en algunos estudios sobre la obra de Iván Efremov se ha querido ver la existencia de aspectos y de concepciones de corte ocultista y disidentes de la ideología soviética (Nudelman, 1989, p. 55), en El corazón de la serpiente se hace evidente el uso de la teoría marxista respecto a la realidad histórico-social y la relación entre materia y conciencia. De esta forma hace una labor propagandista a favor del comunismo, pero también una difusión de aspectos de la teoría marxista.

La oposición entre sistema capitalista y socialista hace de esta obra una expresión de la Guerra Fría, no obstante, la base de la defensa del socialismo apela más a aspectos de orden teórico que a la oposición de sistemas de valores culturales y religiosos como propone Nudelman (1989, p.59) Esto permite la reflexión teórica sobre los fundamentos de la transformación de la realidad histórico-social, lo que conlleva una reflexión sobre los modos de imaginar la sociedad futura con base en la teoría de los modos de producción y la ley de la correspondencia entre las fuerzas productivas y as relaciones de producción. Sumado a esto, al aplicar la ley de la determinación de la base material sobre la superestructura, plantea la forma "científica" en que se debe imaginar la sociedad futura.

El conjunto de estas reflexiones, llevan a afirmar que para Efremov la ciencia ficción del mundo capitalista parte de ideas erróneas y no científicas del futuro de la sociedad, por lo que traspalan aspectos ideológicos del capitalismo al futuro y a los encuentros extraterrestres, tales 
como la agresión y guerra. Al mismo tiempo Efremov en su novela da un ejemplo de las formas en que se debe prefigurar el encuentro interplanetario y la sociedad futura. Esto lleva a que Efremov planee la posibilidad de la existencia de una ciencia ficción dura, en sentido que se basa en la ciencia de la historia, y una ciencia ficción blanda que estaría basada en la "ideología burguesa".

Esto plantea una serie de problemáticas para la teoría literaria. La ciencia ficción de Efremov, en específico El corazón de la serpiente, responde a postulados de la ciencia de la historia, pero no deja de ser una novela de ciencia ficción. Por tanto, se puede concluir que dicha obra está a caballo entre la ciencia de la historia y la ciencia ficción. Esta propuesta nuevamente da aspectos para que la teoría literaria indague sobre los límites entre literatura e historia, en este caso, los linderos entre la ciencia de la historia y la ciencia ficción.

\section{Referencias}

Arjiptsev, F. (1966). La materia como categoría filosófica. México: Grijalbo.

Barrett, T. (2012). Heart of a Serpent? The Cold War Science Fiction of Murray Leinster. Science Fiction Studies, Vol. 39, No. 2, 95-220.

Brandis, E. (1950). Sovetskii nauchno-fantasticheskii roman. Leningrad: Nauka.

Bunge, M. (1947). La ciencia soviética vista por los sabios británicos. La Plata: Editorial Calomino.

Efremov. I. (S/F). El corazón de la serpiente. Moscú:

Grebens (1978), Ivan Efremov's Theory of Soviet Science Fiction. Nueva York: Vantage Press.

Konstantinov, F. (1956) El materialismo histórico. México: Grijalbo.

Lenin, V. (1970), El romanticismo económico, México: Grijalbo.

Marx, C. (1970). Prólogo a la contribución de la crítica de la economía política. En Marx, C y Engels, F. El capital visto por su autor (p. 37-43), México, Grijalbo.

Marx, C y Engels, F. (1972). Manifiesto del Partido Comunista. México: Editorial Roca.

Nudelman R. (1989). Soviet Science Fiction and the Ideology of Soviet Society. Science Fiction Studies, Vol. 16, No. 1, 38-66. 
Rullkotter, B. (1974). He depiction of the hero in soviet russian scientific fantasy. Aspects of alienation in a peripheral genre. Tesis para obtener el grado de maestro por la University of Glasgow.

Schettino, E. (1993). Teoría de la historia. México, UNAM, 1993.

Schwartz, M. (2011). Guests from Outer Space. Occult Aspects of Soviet Science Fiction. En Birgit M., Hagemeister. M., Rosenthal B. (Ed.). (2011). The New Age of Russia Occult and Esoteric Dimensions.(211-237), Berlín: Verlag Otto Sagner.

Shorojova, E. (1979). Fundamentos fisiológicos de la conciencia. México: Grijalbo Strugatski, A., Strugatski, B. (1970). Qué difícil es ser Dios. Moscú: MIR.

Suvin D. (1971). The Utopian Tradition of Russian Science Fiction. The Modern Language Review, Vol. 66, No. 1, 139-159.

Vólkov. G. (1975). El hombre y la revolución científico-técnica. Moscú: Editorial Progreso

Tucker, F. (1974). Recent Development and Outlook". The Russian Review, Vol. 33, No. 2, 189-200. 